\title{
Structural maintenance of chromosomes 2 is identified as an oncogene in bladder cancer in vitro and in vivo
}

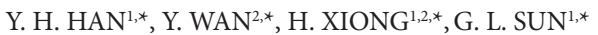 \\ ${ }^{1}$ Laboratory for Medical Research, Department of Clinical Medicine, Tibet University Medical College, Lhasa, China; ${ }^{2}$ Department of Geriatric \\ Medicine and Neurology, West China School of Public Health and West China Forth Hospital, Sichuan University \\ *Correspondence: ionghuahan@163.com; wanyang2014@scu.edu.cn; xhxysq@126.com; guiliisun@163.com
}

Received May 10, 2019 / Accepted August 26, 2019

\begin{abstract}
Taurine upregulated gene 1 (TUG1) has been found to promote bladder cancer cell growth in our recent research. In this study, TUG1-depleted bladder cancer cells were used to identify potent players in bladder cancer. Human gene expression arrays were used for transcriptome profiling of TUG1-depleted bladder cancer cells. Cell proliferation was analyzed by MTT assay. Cell apoptosis and cell cycle were analyzed by flow cytometry. Colony formation assay was used to observe the changes of colony formation rates. Xenograft formation assay was performed in nude mice. Immunohistochemical staining was used to test the gene expression levels in tissues from bladder cancer patients. We found that deregulated genes were strongly enriched in cell cycle or pathways in cancer in TUG1-depleted bladder cancer cells. Structural maintenance of chromosomes 2 (SMC2) was inhibited after TUG1 knockdown. The depletion of TUG1 or SMC2 led to G2/M phase arrest in bladder cancer cells. SMC2 depletion inhibited bladder cancer cell proliferation, promoted apoptosis, decreased colony formation, and reduced tumor growth in xenograft nude mice. Overexpression of SMC2 restored the growth of TUG1-depleted cells. The expression levels of SMC2 were higher in human bladder cancer tissues than that in paired normal tissues. Our data suggest that SMC2 is an oncogene in bladder cancer and depletion of SMC2 might have potential therapeutical significance in bladder cancer.
\end{abstract}

Key words: bladder cancer, structural maintenance of chromosomes 2, oncogene

Bladder cancer remains a big threat to human health globally today. An estimated 79,030 new bladder cancer cases and 16,870 bladder cancer deaths are reported for 2017 in the United States [1]. The molecular mechanisms underlying the disease are rather complicated [2] and understudied. Long intergenic non-coding RNAs (lincRNAs) are expressed in human cells. They co-work with chromatin modifying complexes to regulate gene expression [3]. Taurine upregulated gene 1 (TUG1) is a lincRNA that is upregulated in bladder cancer. The depletion of TUG1 induces proliferation inhibition and apoptosis of bladder cancer cells [4]. These findings suggest that TUG1 plays a proliferative role in bladder cancer. But it remains unknown how TUG1 regulates the transcriptome of bladder cancer.

This study profiled the transcriptome of TUG1-depleted bladder cancer cells. We found that 209 genes were upregulated and 223 were downregulated after TUG1 depletion. Deregulated genes were strongly enriched in cell cycle or pathways in cancer. The expression of Structural maintenance of chromosomes 2 (SMC2) was significantly inhibited after TUG1 depletion. The expression levels of SMC2 were higher in human cancerous tissues than that in paired normal tissues. In vitro and in vivo assays identified that SMC2 was an oncogenic player in bladder cancer.

\section{Materials and methods}

Cell lines and infections. Bladder cancer cell lines T24 and 5637 in this study were obtained from the Institute of Cell Research, Chinese Academy of Sciences, Shanghai, China. The cells were cultured as previously documented [5]. Cells were infected with TUG1 shRNA, SMC2 shRNA, SMC2 ORF or negative control lentiviruses.

RNA extraction and qRT-PCR analysis. The procedures for RNA extraction from the cultured cells and qRT-PCR analyses for target genes were performed as previously described [5].

shRNA target sequences and primer sequences. TUG1 shRNA target sequence was 5'-CACGACCATGGTTGTCATCCA-3'. SMC2 shRNA target sequence was 5'-AGATT- 
TACTCAATGTCAAA-3'. The forward and reverse primers for TUG1 were 5'-TAGCAGTTCCCCAATCCTTG-3' and 5'-CACAAATTCCCATCATTCCC-3', respectively. The forward and reverse primers for SMC2 were 5'-ACAACACCAGAGTACAGGATC-3' and 5'-CGGCCCTGCATGATGAGAA-3', respectively. The forward and reverse primers for GAPDH were 5'-TGACTTCAACAGCGACACCCA-3' and 5'-CACCCTGTTGCTGTAGCCAAA-3',

respectively. GAPDH was taken as an internal control for TUG1 and SMC2. Other shRNA target sequences for TUG1 and SMC2 had been investigated in our pre-experiment. The panel of shRNA target sequences used in the pre-experiment is provided in Supplementary Table S1.

Transcriptome microarray analysis. T24 cells were infected with either TUG1 shRNA lentiviruses or negative control lentiviruses. Affymetrix GeneChip ${ }^{\otimes}$ PrimeView $^{\mathrm{TM}}$ Human Gene Expression Arrays (Affymetrix, Santa Clara, CA, USA) were used for transcriptome profiling of TUG1-depleted bladder cancer cells according to the manufacturer's instructions. Microarray analysis was done in triplicate.

Proliferation, cell cycle, colony formation and apoptosis assays. Cell proliferation by the 3-(4,5-dimethylthiazol-2yl)-2,5-diphenyl tetrazolium bromide (MTT) assay, cell cycle analysis by flow cytometry and colony formation assay were used as described elsewhere [6]. Cell apoptosis was analyzed by flow cytometry as previously described [7].

Xenograft formation assay. Four-week-old female $\mathrm{BALB} / \mathrm{c}$ nude mice were obtained from Shanghai Lingchang Biolotech co. Ltd. Shanghai, China. The use of mice was approved by the Institutional Animal Care and Use Committee of Tibet University Medical College, Lhasa, China. Ten nude mice were injected into the right flank with $1 \times 10^{7}$ T24 cells infected with SMC2 shRNA lentiviruses as an experimental group. Another 10 nude mice were injected into the right flank with $1 \times 10^{7} \mathrm{~T} 24$ cells infected with control lentiviruses as a control group. The mice were sacrificed and the tumors were weighed 36 days after the injection.

Patient samples. Formalin-fixed paraffin-embedded cancer tissues and paired normal tissues from 31 bladder cancer patients were acquired from Shanghai Outdo Biotech Co. Ltd., Shanghai, China. The tissues were obtained with informed consent from all individuals. The clinicopathological characteristics of the patients are provided in Supplementary Table S2. The use of patient samples in the study was approved by the Institutional Review Board of Tibet University Medical College, Lhasa, China.

Immunohistochemical staining methods were used as described elsewhere [8]. The scores of staining intensity were scaled as 0 (no staining), 1 (weak staining), 2 (moderate staining) and 3 (strong staining). The scores of staining rate were scaled as 1 (1-25\%), 2 (26-50\%), 3 (51-75\%) and 4 (76-100\%). The total score equals the score of staining intensity $\times$ the score of the staining rate. The total score $\leq 4$ was defined as low expression and the total score $>4$ score was defined as high expression.

Statistical analyses The differences of SMC2 expression levels between human cancerous tissues and paired normal tissues were determined by sign-test. MTT assays were analyzed by the analysis of variance. Differences in gene expression changes in bladder cancer cells after infection, apoptosis, colony formation, cell cycle and xenograft formation assays were analyzed by Student's t-test. Data were shown as mean \pm SD. A $\mathrm{p}<0.05$ was considered statistically significant.

\section{Results}

Transcriptome profiling of TUG1-depleted bladder cancer cells. Forty-eight hours after infection with TUG1 shRNA lentiviruses or negative control lentiviruses, the TUG1 RNA expression levels were measured. The inhibitory rates (TUG1 shRNA / negative control) were $84.54 \pm 1.76 \%$ and $78.23 \pm 1.33 \%$ in T24 and 5637 cells, respectively. To identify vital players in bladder cancer that are regulated by TUG1, we profiled the transcriptome of TUG1-depleted bladder cancer T24 cells using Affymetrix GeneChip ${ }^{\oplus}$ PrimeView ${ }^{\mathrm{TM}}$ Human Gene Expression Arrays. The expression levels of genes in TUG1-depleted T24 cells were compared with that in control T24 cells. Over hundreds of genes were deregulated after TUG1 depletion. TUG1-depleted T24 cells showed distinct transcriptomic patterns compared with control T24 cells. The impact of TUG1 depletion in T24 cells on transcriptomics demonstrated by heatmap is provided in Supplementary Figure S1. In this study, statistically significant differential expression levels were defined as $\mid$ Fold Change $\mid>1.5$, p-value $<0.05$. We found that 209 genes were upregulated and 223 were downregulated after TUG1 depletion. With these deregulated genes, pathway enrichment analysis was performed. Deregulated genes were strongly enriched in KEGG cell cycle, KEGG pathways in cancer, BIOCARTA caspase pathway, etc. The pathways analyzed with the top ten $-\log _{10}$ (p-value) values are shown in Supplementary Figure S2.

TUG1 depletion inhibited SMC2 expression in bladder cancer cells. To identify possible novel players in bladder cancer, primary screening based on available biological information from the public domain was performed. Deregulated genes that were rarely documented and might play proliferative or anti-proliferative roles in cancer were chosen from the panel of 209 upregulated genes and 223 downregulated genes in TUG1 depleted bladder cancer cells as candidates for further study. SMC2 was one candidate that was downregulated after TUG1 depletion. Forty-eight hours after infection with TUG1 shRNA or negative control lentiviruses, the SMC2 RNA expression levels in T24 and 5637 cells were measured. The SMC2 mRNA expression inhibitory rates caused by TUG1 depletion (TUG1 shRNA / negative control) were $76.2 \pm 2.79 \%$ and $73.8 \pm 3.55 \%$ in T24 and 5637 cells, respectively. 
Impact of SMC2 on proliferation, apoptosis and colony formation of bladder cancer cells. Forty-eight hours after infection with SMC2 shRNA or negative control lentiviruses, the SMC2 mRNA expression levels in T24 and 5637 cells were measured. The SMC2 mRNA expression inhibitory rates (SMC2 shRNA / negative control) were $81.8 \pm 3.12 \%$ and $80.2 \pm 1.27 \%$ in T24 and 5637 cells, respectively (Figures 1A, $1 \mathrm{C})$. To investigate the impact of SMC2 on the proliferation of bladder cancer cells, MTT assays were used to measure the cell proliferation changes of T24 and 5637 cells after SMC2 knockdown. Cell proliferation inhibition after SMC2 knockdown was observed in both T24 cells (Figure 1B) and 5637 cells (Figure 1D) by MTT assay. Seventy-two hours after infection, the cell apoptosis changes were determined by Annexin $\mathrm{V}$ apoptosis detection by flow cytometry analysis. The depletion of SMC2 promoted apoptosis of T24 cells (Figure 2A) and 5637 cells (Figure 2B), respectively. The impact of SMC2 on the colony formation was measured. Seventy-two hours after infection, the cells were seeded and cultured for 11 days. Decreased colony formation numbers caused by SMC2 knockdowns were observed in both T24 cells (Figure 2C) and 5637 cells (Figure 2D).

Depletion of TUG1 or SMC2 led to G2/M phase arrest in bladder cancer cells. We investigated the impact of TUG1 and SMC2 on cell cycle. T24 cells were cultured, infected, collected and stained with propidium iodide. Cell cycle analysis was performed. Increased percentages of cells were observed in G2/M phase of TUG1 KD or SMC2 KD group
(Figure 3). The experiments are supportive of the findings that deregulated genes were strongly enriched in the cell cycle in TUG1-depleted bladder cancer cells and SMC2 acted as a downstream player of TUG1.

Depletion of SMC2 reduced tumor growth in xenograft nude mice. The mice were sacrificed and the tumors were weighed 36 days after the injection of T24 cells infected with SMC2 shRNA or control lentiviruses. SMC2 knockdown reduced tumor growth in xenograft nude mice $(\mathrm{p}=0.0191)$ (Figure 4).

Overexpression of SMC2 restored the proliferation of SMC2-depleted or TUG1-depleted bladder cancer cells. To demonstrate the role of SMC2 in promoting the proliferation of bladder cancer cells, the overexpression of SMC2 was done to test the impact of proliferation on SMC2-depleted T24 cells. Forty-eight hours after infection with SMC2 shRNA lentiviruses or SMC2 shRNA plus SMC2 ORF lentiviruses, the SMC2 mRNA expression levels were measured. Overexpression of SMC2 increased the SMC2 mRNA expression levels in SMC2-depleted T24 cells significantly (Figure 5A). Cell proliferation promotion caused by overexpression of SMC2 was observed in SMC2-depleted T24 cells (Figure 5B). To corroborate that SMC2 acted downstream of TUG1, overexpression of SMC2 was done to test the impact of proliferation on TUG1-depleted bladder cancer cells. Fortyeight hours after infection with TUG1 shRNA lentiviruses or TUG1 shRNA plus SMC2 ORF lentiviruses, the SMC2 mRNA expression levels were measured. Overexpression
A

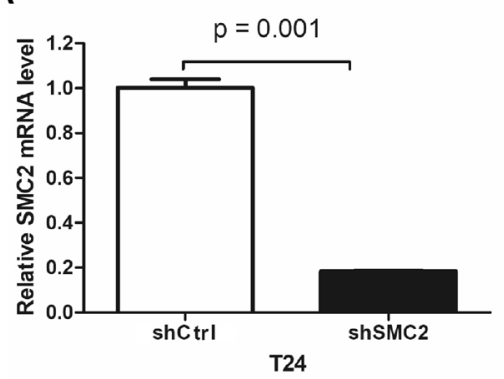

C

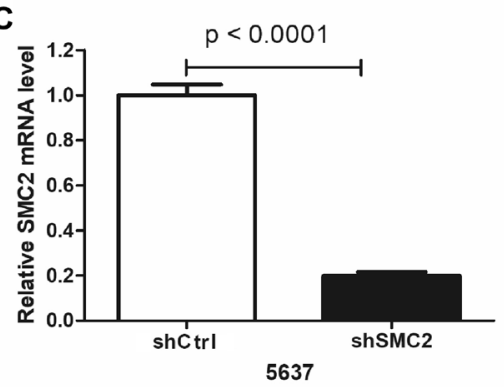

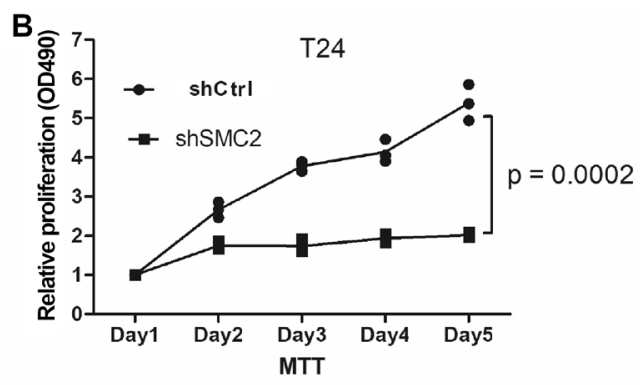

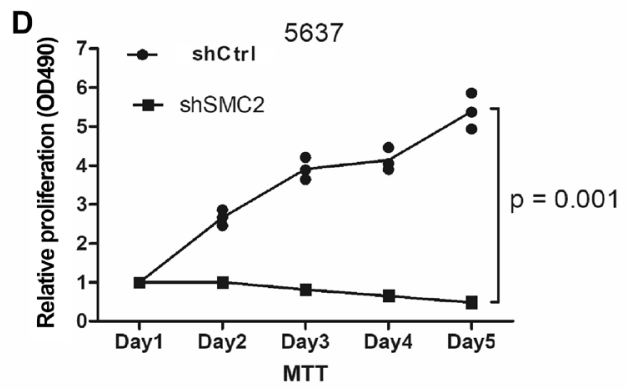

Figure 1. The impact of SMC2 on the proliferation of bladder cancer cells. SMC2 knockdown inhibited SMC2 expression significantly in T24 (A) and 5637 (C) cells. Real-Time qPCR was used to test SMC2 mRNA expression levels. The comparative $\Delta$ Ct method (value of $2^{-\Delta C t(S M C 2}$ - GAPDH) was used to show the mRNA expression levels. Each experiment of expression assay was done in 3 replicates. Data are shown as mean \pm SD. Cell proliferation inhibition caused by SMC2 knockdown was observed in T24 (B) and 5637 (D) cells. Cell proliferation was measured by MTT assay. Each experiment of MTT assay in both cell lines was performed in 5 replicates. Data are shown as mean \pm SD. shCtrl stands for the control group. shSMC2 stands for the SMC2 knockdown group. 

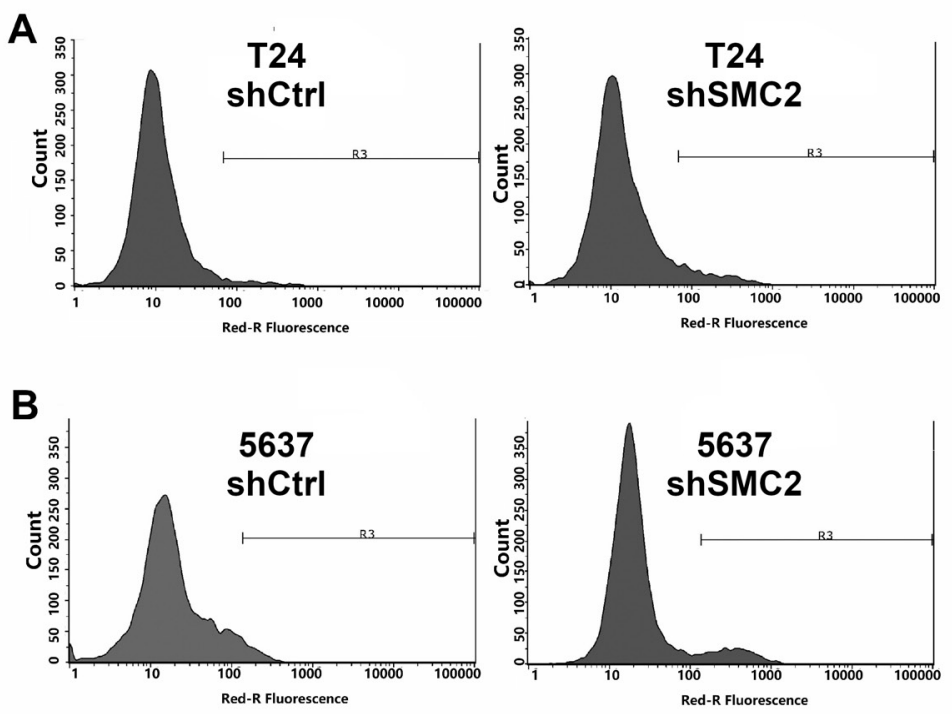

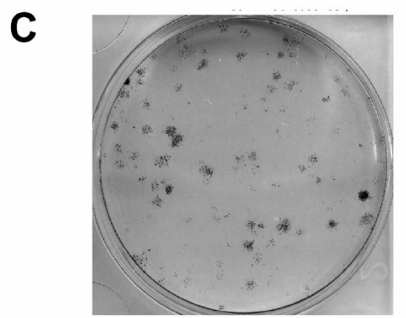

T24 shCtrl

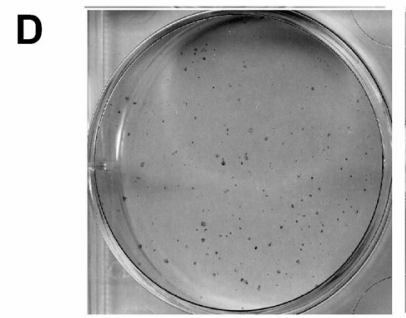

5637 shCtrl

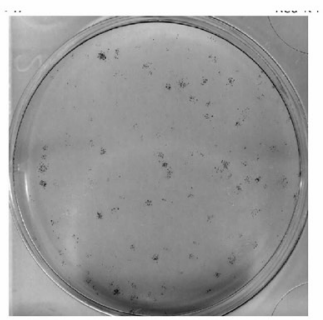

T24 shSMC2

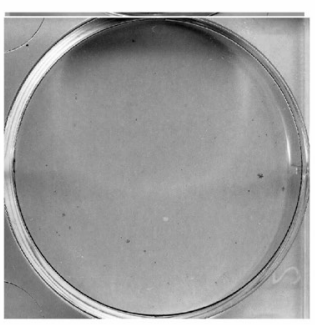

5637 shSMC2
T24
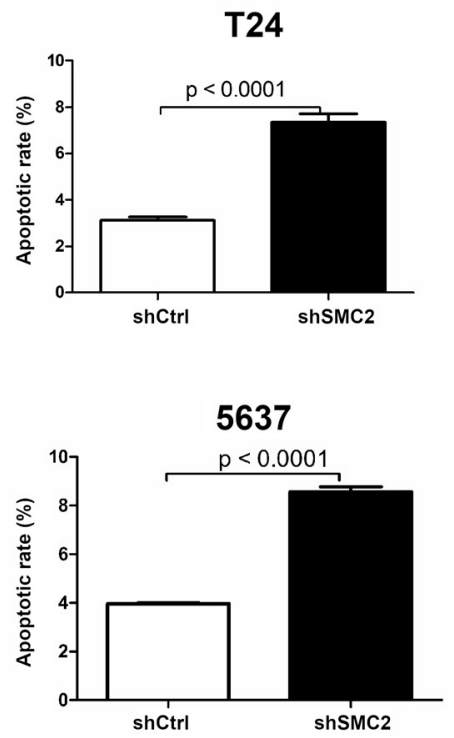

T24

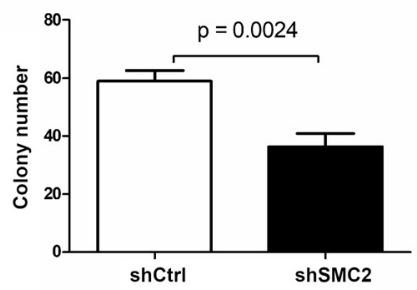

5637

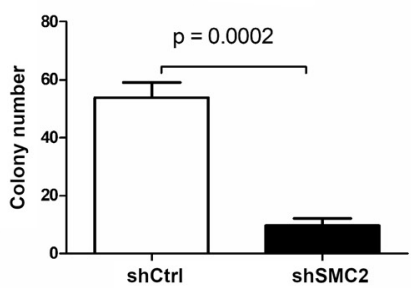

Figure 2. The impact of SMC2 on the apoptosis and colony formation of bladder cancer cells. shCtrl stands for the control group. shSMC2 stands for the SMC2 knockdown group. Seventy-two hours after infection with SMC2 shRNA or negative control lentiviruses, the cell apoptosis changes were determined by flow cytometry analysis. Each experiment of flow cytometry assay in both cell lines was performed in triple. Data are shown as mean \pm SD. A) Representative images of flow cytometry analysis in the control group and the SMC2 knockdown group in T24 cells. Cell apoptosis caused by SMC2 knockdown was observed in T24 cells. B) Representative images of flow cytometry analysis in control group and SMC2 knockdown group in 5637 cells. Cell apoptosis caused by SMC2 knockdown was observed in 5637 cells. Seventy-two hours after infection with SMC2 shRNA or negative control lentiviruses, the cells were seeded and cultured for 11 days. The culture medium was replaced every 2 days. Each experiment of colony formation assay in both cell lines was performed in triplicate. Data are shown as mean \pm SD. C) Representative images of colony formation in the control group and the SMC2 knockdown group in T24 cells. Decreased colony formation numbers caused by SMC2 knockdowns were observed in T24 cells. D) Representative images of colony formation in the control group and the SMC2 knockdown group in 5637 cells. Decreased colony formation numbers caused by SMC2 knockdown were observed in 5637 cells.

of SMC2 increased the SMC2 mRNA expression levels in TUG1-depleted T24 cells markedly (Figure 5C). Cell proliferation promotion caused by overexpression of SMC2 was observed in TUG1-depleted T24 cells (Figure 5D).
Upregulation of SMC2 in bladder cancerous tissues The expression levels SMC2 were immunohistologically tested in 31 patients with bladder cancerous tissues and paired normal tissues. The expression levels of SMC2 were 

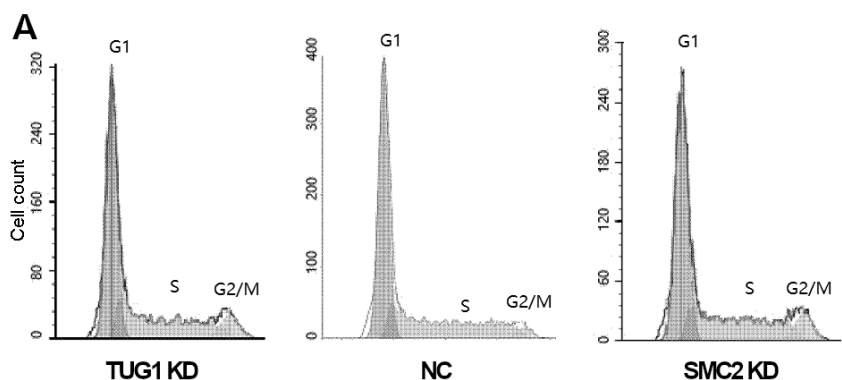

B

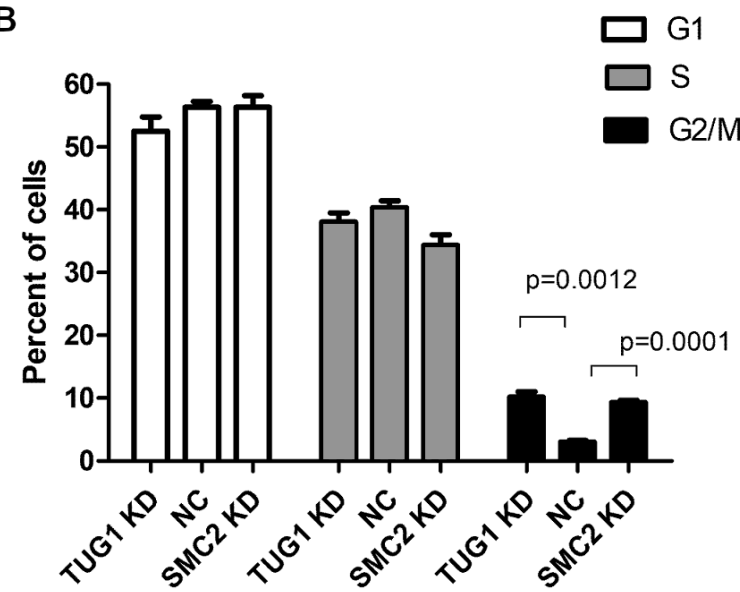

Figure 3. The depletion of TUG1 or SMC2 led to G2/M phase arrest in bladder cancer cells. Bladder cancer T24 cells were stained with propidium iodide. Cell cycle analysis was performed by flow cytometry. NC stands for the control group. KD stands for the knockdown group. G1 stands for G1 phase. S stands for S phase. G2 stands for G2 phase. M stands for M phase. A) Representative images of flow cytometry. B) Increased percentages of cells were observed in G2/M phase of TUG1 KD and SMC2 KD group.

higher in cancerous tissues than that in paired normal tissues $(\mathrm{p}=0.002$, Figure 6$)$.

\section{Discussion}

LincRNAs constitute a large family of long non-coding RNAs that regulate gene expression via chromatin remodeling. Nearly 3,300 lincRNAs are identified in human cells. TUG1 is a lincRNA that modifies the chromatin state to affect the expression of sets of genes [3].

In our recent study, we have found that TUG1 expression levels are upregulated in bladder cancer, higher grade and stage bladder cancers have higher TUG1 expression levels, TUG1 depletion in bladder cancer T24 and 5637 cells inhibits bladder cancer cell proliferation and increases bladder cancer cell apoptosis [4]. But the roles of TUG1 on the transcriptome-wide regulation in bladder cancer cells are not clear.

In this work, we profiled the transcriptome of TUG1-depleted bladder cancer cells. We found that 209
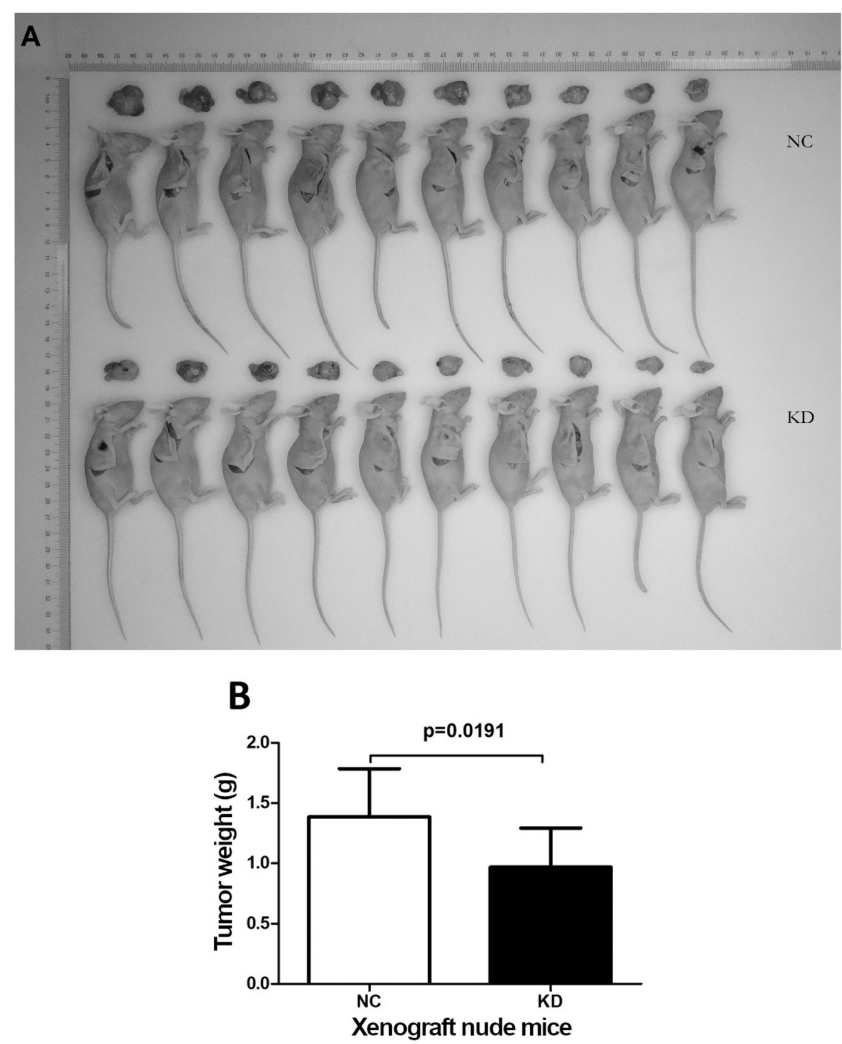

Figure 4. SMC2 knockdown reduced tumor growth in nude mice. NC stands for the control group. KD stands for the SMC2 knockdown group. A) Images of the nude mice and the xenografts of the control group and the SMC2 knockdown group. B) SMC2 knockdown reduced tumor weight in nude mice.

genes were upregulated and 223 were downregulated after TUG1 depletion. Many of these genes are related to the cell cycle or pathways in cancer. These findings help to understand the proliferative role of TUG1 in bladder cancer.

SMC2 protein is a subunit of the human condensin complex [9]. Condensin mutations may cause chromosomal destabilization in lymphoma [10]. SMC2 is upregulated in human colorectal cancer. The depletion of SMC2 reduces tumor growth in colorectal cancer in xenograft nude mice [11], suggesting that the hyperactivity of SMC2 is oncogenic.

In this study, SMC2 was inhibited after TUG1 knockdown in bladder cancer. The depletion of TUG1 or SMC2 led to G2/M phase arrest in T24 cells. The depletion of SMC2 inhibited proliferation, promoted apoptosis and decreased colony formation of bladder cancer cells and reduced tumor growth in xenograft nude mice. Cell proliferation promotion caused by overexpression of SMC2 was observed in SMC2-depleted or TUG1-depleted T24 cells. The expression levels of SMC2 were higher in human bladder cancer tissues than that in 
A

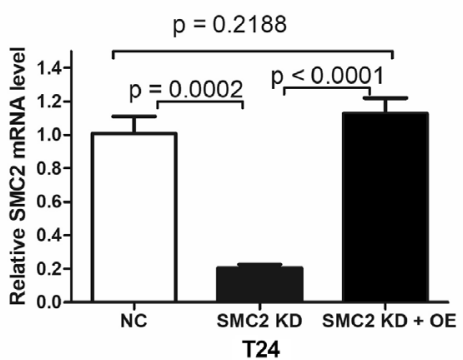

C

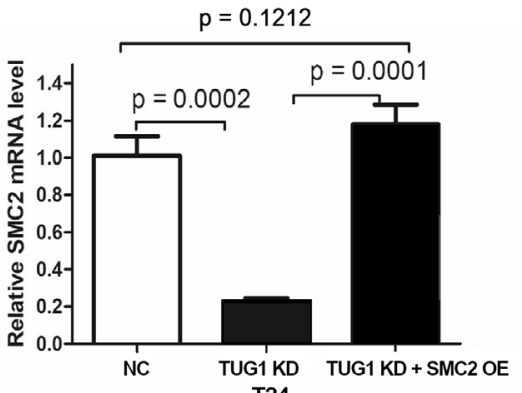

B

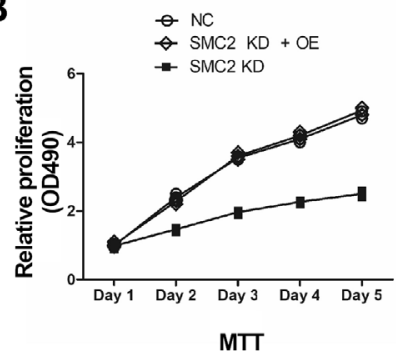

D

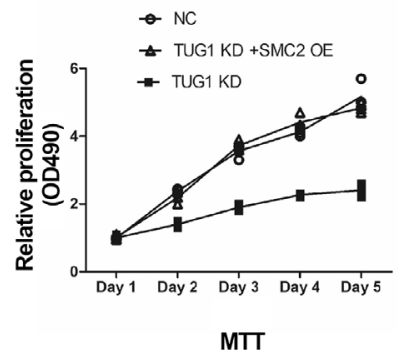

Figure 5. Overexpression of SMC2 restored the proliferation of SMC2-depleted or TUG1-depleted T24 cells. NC stands for the control group, KD stands for the knockdown and $\mathrm{OE}$ stands for the overexpression. Each experiment of expression assay was done in 3 replicates. Data are shown as mean \pm SD. Cell proliferation was measured by MTT assay and each experiment was performed in 5 replicates. ANOVA was used for the comparison of curves of cell proliferation. Data are shown as mean \pm SD. A) SMC2 KD depleted SMC2 in T24 cells (SMC2 KD versus NC). Overexpression of SMC2 increased the SMC2 mRNA expression levels in SMC2-depleted T24 cells significantly (SMC2 KD + OE versus SMC2 KD). Overexpression of SMC2 restored the expression of SMC2 in SMC2-depleted T24 cells (SMC2 KD + OE versus NC). B) SMC2 KD inhibited T24 cell proliferation (SMC2 KD versus NC, $\mathrm{p}<0.0001)$. Cell proliferation promotion caused by overexpression of SMC2 was observed in SMC2-depleted T24 cells (SMC2 KD + OE versus SMC2 KD, p<0.0001). Overexpression of SMC2 restored the proliferation of SMC2-depleted T24 cells $(\mathrm{SMC} 2 \mathrm{KD}+\mathrm{OE}$ versus NC, $\mathrm{p}=\mathbf{0 . 1 7 2 3})$. C) TUG1 KD depleted SMC2 in T24 cells (TUG1 KD versus NC). Overexpression of SMC2 increased the SMC2 mRNA expression levels in TUG1-depleted T24 cells (TUG1 KD + SMC2 OE versus TUG1 KD). Overexpression of SMC2 restored the expression of SMC2 in TUG1-depleted T24 cells (TUG1 KD + SMC2 OE versus NC). D) TUG1 KD inhibited T24 cell proliferation (TUG1 KD versus NC, $p<0.0001$ ). Cell proliferation promotion caused by overexpression of SMC2 was observed in TUG1-depleted T24 cells (TUG1 KD + SMC2 OE versus TUG1 KD, p $<0.0001$ ). Overexpression of SMC2 restored the proliferation of TUG1-depleted T24 cells (TUG1 KD + SMC2 OE versus NC, $\mathrm{p}=0.1170$ ).

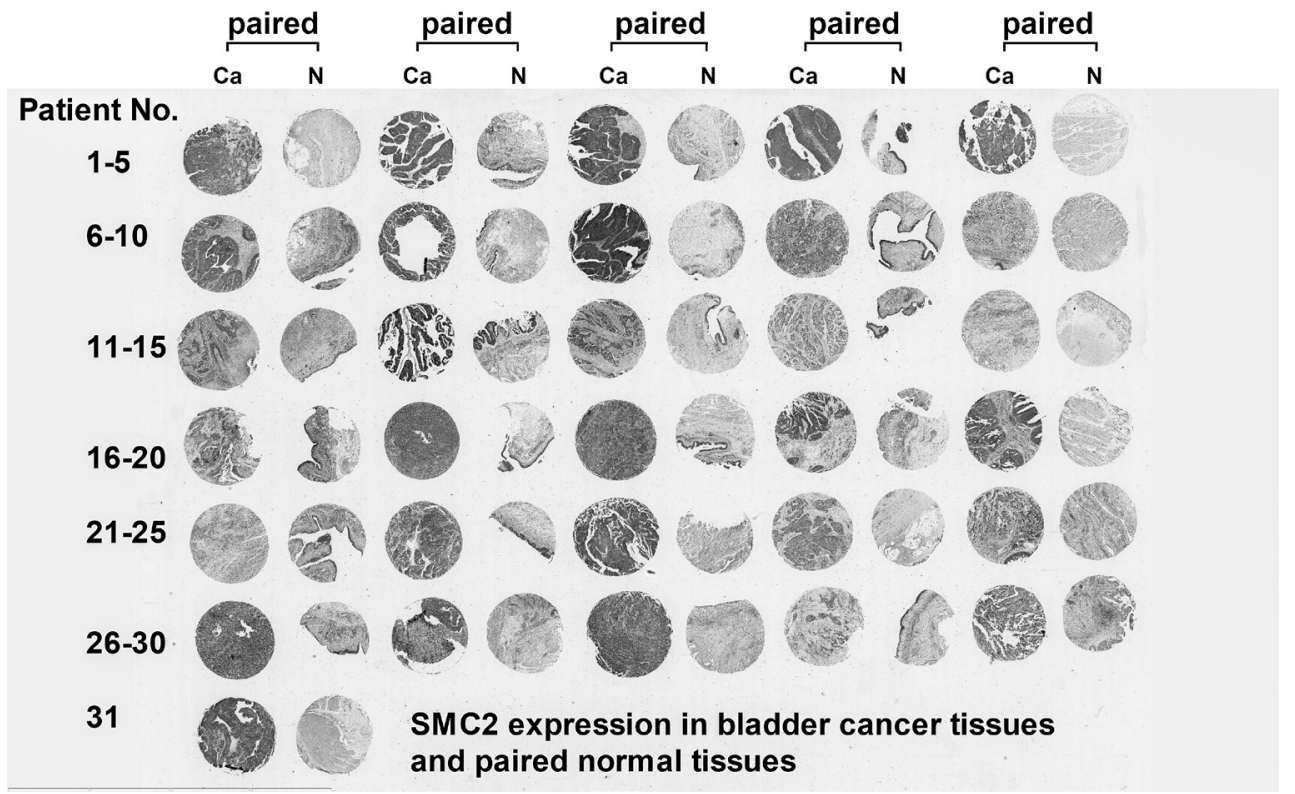

Figure 6. The expression levels of SMC2 in bladder cancer. Ca stands for cancerous tissue. N stands for paired normal tissue. A total of 31 bladder cancer patients were tested immunohistochemically. All the tumors are urothelial carcinomas of the bladder. The expression levels of SMC2 were higher in cancerous tissues than that in paired normal tissues $(\mathrm{p}=\mathbf{0 . 0 0 2})$. 
paired normal tissues. These findings suggest that SMC2 is a TUG1-regulated oncogenic player in bladder cancer.

The above findings help deepen our understanding of the underlying mechanism of bladder cancer, but more work is needed to identify the co-players, the functioning patterns and the powerful downstream actors of SMC2 in promoting the proliferation of bladder cancer cells.

Taken together, SMC2 is a downstream player of TUG1; SMC2 is an oncogene in bladder cancer; silencing SMC2 might be a promising intervention alternative for bladder cancer.

Supplementary information is available in the online version of the paper.

Acknowledgments: This work was supported by the National Natural Science Foundation of China [Grant number: 81372735], the Scientific Research Foundation for the Introduction of Talents of Tibet University [Grant number: 2018-6], the Special Fund for Human Resources Development of Tibet Autonomous Region [Grant number: 2018-47], the Foundation for Key Disciplines of Higher Schools from the Department of Education of Tibet Autonomous Region [Grant number: 2017-49], the Science and Technology Support Program of Sichuan Province [2011SZ0090], the Natural Science Foundation of Tibet Autonomous Region [2016ZR-TU-06], the Training Foundation of Tibet University [ZDTSJH18-09], the Young Scientist Fund of the National Natural Science Foundation of China [81301795], the Innovation Project for Young Medical Researchers of Sichuan Medical Association [Q16054], and the Scientific Research Foundation of West China Forth Hospital [2014syqd027].

\section{References}

[1] SIEGEL RL, MILLER KD, A. JEMAL A. Cancer Statistics. CA Cancer J Clin 2017; 67: 7-30. https://doi.org/10.3322/ caac. 21387

[2] PANNEERSELVAM J, PARK HK, ZHANG J, DUDIMAH FD, ZHANG P et al. FAVL impairment of the Fanconi anemia pathway promotes the development of human bladder cancer. Cell Cycle 2012; 11: 2947-2955. https://doi. org/10.4161/cc. 21400
[3] KHALIL AM, GUTTMAN M, HUARTE M, GARBER M, RAJ A et al. Many human large intergenic noncoding RNAs associate with chromatin-modifying complexes and affect gene expression. Proc Natl Acad Sci U S A 2009; 106: $11667-$ 11672. https://doi.org/10.1073/pnas.0904715106

[4] HAN Y, LIU Y, GUI Y, CAI Z. Long intergenic non-coding RNA TUG1 is overexpressed in urothelial carcinoma of the bladder. J Surg Oncol 2013; 107: 555-559. https://doi. org/10.1002/jso.23264

[5] HAN Y, LIU Y, ZHANG H, WANG T, DIAO R et al. HsamiR-125b suppresses bladder cancer development by downregulating oncogene SIRT7 and oncogenic long non-coding RNA MALAT1. FEBS Lett 2013; 587: 3875-3882.

[6] SHEN B, ZHANG Y, YU S, YUAN Y, ZHONG Y et al. MicroRNA-339, an epigenetic modulating target is involved in human gastric carcinogenesis through targeting NOVA1. FEBS Lett 2015; 589: 3205-3211. https://doi.org/10.1016/j. febslet.2015.09.009

[7] HAN Y, LIU Y, NIE L, GUI Y, CAI Z. Inducing cell proliferation inhibition, apoptosis, and motility reduction by silencing long noncoding ribonucleic acid metastasis-associated lung adenocarcinoma transcript 1 in urothelial carcinoma of the bladder. Urology 2013; 81: 209.e1-7. https://doi. org/10.1016/j.urology.2012.08.044

[8] HUANG Y, FANG C, SHI JW, WEN Y, LIU D. Identification of hMex-3A and its effect on human bladder cancer cell proliferation. Oncotarget 2017; 8: 61215-61225. https://doi. org/10.18632/oncotarget. 18050

[9] HUDSON DF, MARSHALL KM, EARNSHAW WC. Condensin: Architect of mitotic chromosomes. Chromosome Res 2009; 17: 131-144. https://doi.org/10.1007/s10577-0089009-7

[10] HAM MF, TAKAKUWA T, RAHADIANI N, TRESNASARI K, NAKAJIMA $\mathrm{H}$ et al. Condensin mutations and abnormal chromosomal structures in pyothorax-associated lymphoma. Cancer Sci 2007; 98: 1041-1047. https://doi. org/10.1111/j.1349-7006.2007.00500.x

[11] DAVALOS V, SUAREZ-LOPEZ L, CASTANO J, MESSENT A, ABASOLO I et al. Human SMC2 protein, a core subunit of human condensin complex, is a novel transcriptional target of the WNT signaling pathway and a new therapeutic target. J Biol Chem 2012; 287: 43472-43481. https://doi. org/10.1074/jbc.M112.428466 


\section{Structural maintenance of chromosomes 2 is identified as an oncogene in bladder cancer in vitro and in vivo}

Y. H. $\operatorname{HAN}^{1, *}$, Y. WAN ${ }^{2, *}$, H. XIONG ${ }^{1,2, *}$, G. L. SUN ${ }^{1, *}$

\section{Supplementary Information}

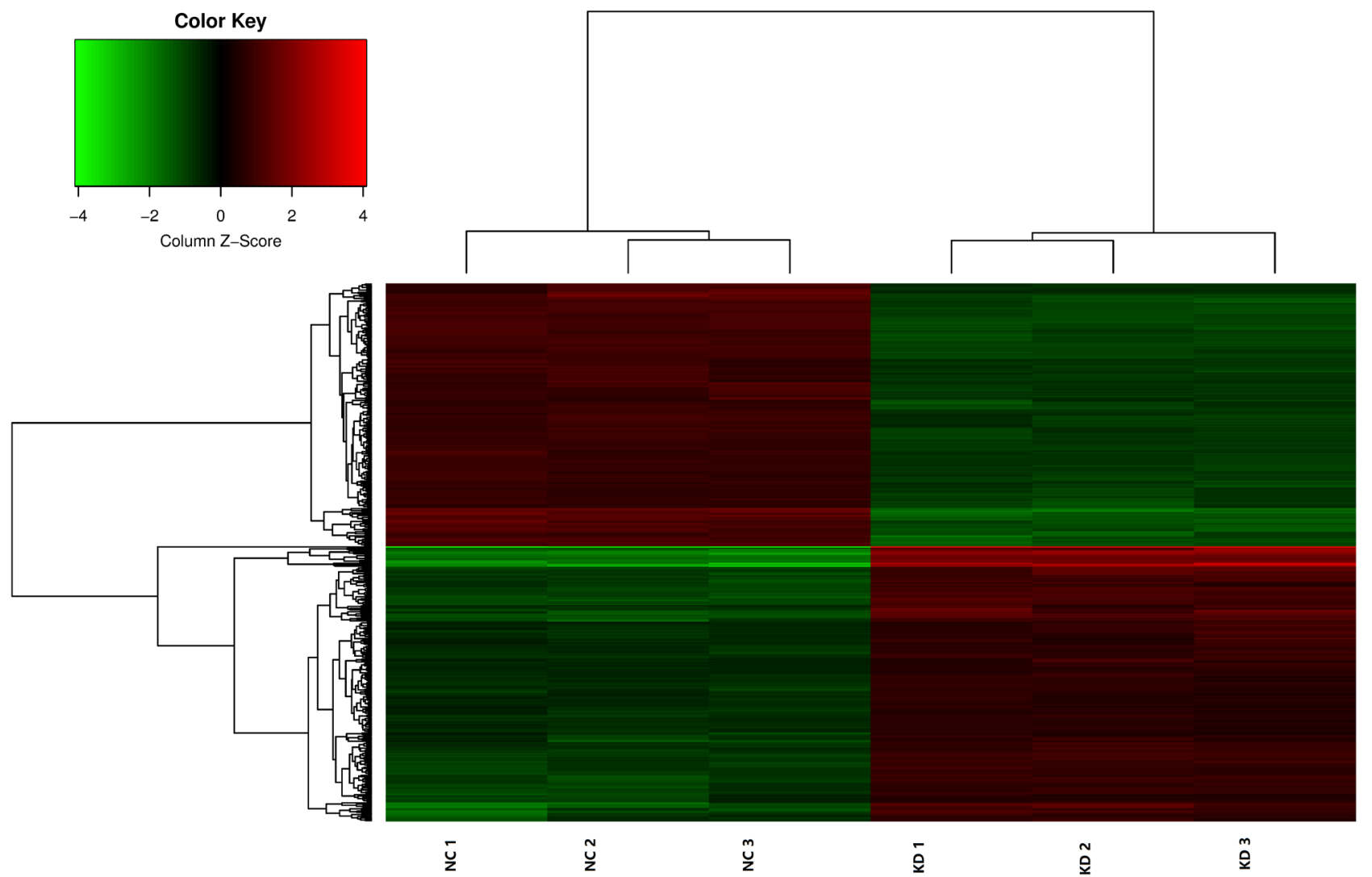

Figure S1. The impact of TUG1 depletion in T24 cells on transcriptomics demonstrated by heatmap. Microarray analysis was done in 3 replicates. NC stands for negative control. KD NC stands for TUG1 knockdown. Numbers 1, 2, 3 are No.s of the replicates. We compared the expression levels of genes in TUG1-depleted T24 cells compared with that in control T24 cells. TUG1-depleted T24 cells showed distinct transcriptomic patterns compared with control T24 cells.

Supplementary Table S1. shRNA target sequences in the pre-exprement.

\begin{tabular}{lll}
\hline Gene & No. & Target Sequence \\
\hline TUG1 $^{*}$ & a $^{\text {s }}$ & 5'-CACGACCATGGTTGTCATCCA-3' \\
& b & 5'-GCCATTTAAAGAAACAGTACC-3' \\
& c & 5'-GGCTATCAGAATAACCACTCA-3' \\
SMC2 $^{*}$ & d $^{\text {s }}$ & 5'-AGATTTACTCAATGTCAAA-3' \\
& e & 5'-GCAAAGTCCTTTAGGATTTGA-3' \\
& f & 5'-GCTCTTTCCTTGGTTGAATAT-3' \\
\hline
\end{tabular}

${ }^{*}$ Inhibition rates in T24 cells. $\mathrm{A}>80 \%$; $, \mathrm{c}, 1 / 2(\mathrm{a}+\mathrm{b}), 1 / 2(\mathrm{a}+\mathrm{c}), 1 / 2(\mathrm{~b}+\mathrm{c})$ : 58\%-74\%. "Inhibition rates in T24 cells. D >80\%; e, f, $1 / 2(\mathrm{~d}+\mathrm{e})$,

$1 / 2(\mathrm{~d}+\mathrm{f}), 1 / 2(\mathrm{e}+\mathrm{f}): 62 \%-76 \%{ }^{\circ}$ The shRNA target sequences showed the highest inhibition rate for each gene, which were chosen for the formal experiments in the Manuscript. 


\section{Pathway Enrichment}

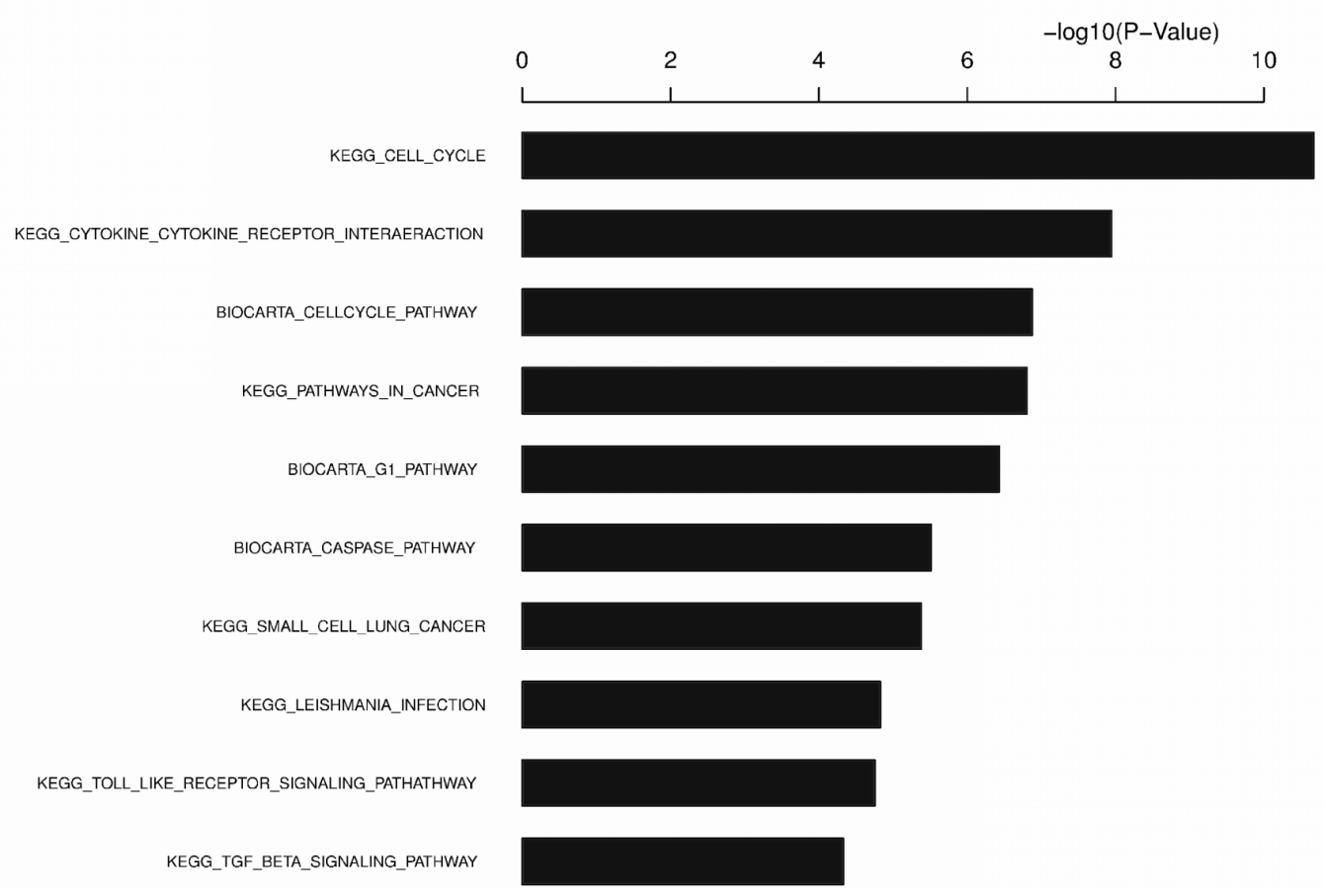

Figure S2. Pathway enrichment analysis in TUG1-depleted T24 cells. We profiled the transcriptome of TUG1-depleted T24 cells and control T24 cells using Affymetrix GeneChip ${ }^{\circ}$ PrimeView ${ }^{\text {Tn }}$ Human Gene Expression Arrays. The expression levels of genes in TUG1-depleted T24 cells and in control T24 cells were compared. The deregulated genes were enriched for pathway analysis. The pathways analyzed with the top ten -log10(p-value) values are shown.

Supplementary Table S2. The clinicopathological characteristics of the patients.

\begin{tabular}{lccccccccc}
\hline No. & Sex & Age & Grade & Stage & No. & Sex & Age & Grade & Stage \\
\hline 1 & Female & 73 & Low & invasive & 17 & Male & 64 & High & invasive \\
2 & Male & 52 & Low & non-invasive & 18 & Male & 75 & High & invasive \\
3 & Male & 77 & Low & non-invasive & 19 & Female & 66 & High & invasive \\
4 & Male & 65 & Low & non-invasive & 20 & Female & 48 & High & invasive \\
5 & Male & 67 & Low & non-invasive & 21 & Male & 67 & High & invasive \\
6 & Male & 61 & Low & non-invasive & 22 & Male & 72 & High & invasive \\
7 & Male & 75 & Low & non-invasive & 23 & Male & 60 & High & invasive \\
8 & Male & 73 & High & non-invasive & 24 & Male & 68 & High & invasive \\
9 & Male & 70 & High & invasive & 25 & Female & 77 & High & invasive \\
10 & Male & 55 & High & invasive & 26 & Male & 57 & High & invasive \\
11 & Male & 76 & High & invasive & 27 & Male & 68 & High & non-invasive \\
12 & Male & 50 & High & invasive & 28 & Male & 55 & High & non-invasive \\
13 & Male & 75 & High & invasive & 29 & Male & 63 & High & non-invasive \\
14 & Male & 74 & High & invasive & 30 & Male & 54 & High & non-invasive \\
15 & Male & 83 & High & invasive & 31 & Male & 62 & High & non-invasive \\
16 & Female & 81 & High & invasive & & & & & \\
\hline
\end{tabular}

No., patient no.. Age, years old. Grade, the 2004 WHO classification. Stage, invasive, muscle-invasive; non-invasive, muscle-non-invasive. All tumors are urothelial carcinomas. 